\title{
Attention and processing of relevant visual information while simulated driving: a MEG study
}

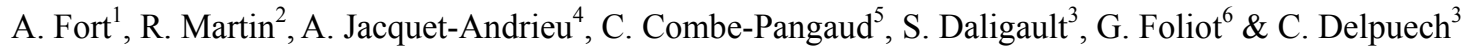 \\ ${ }^{1}$ INRETS-LESCOT, Bron, France \\ ${ }^{2}$ ISH, Lyon, France \\ ${ }^{3}$ CERMEP, Bron, France \\ ${ }^{4}$ Paris Est University / LabInfo - UMR 8049, Paris, France \\ ${ }^{5}$ CRIS/LEACM - EA 647 \\ ${ }^{6}$ ISH/PRI - UMS 1798
}

\begin{abstract}
It is a well-known fact that attention is crucial for driving a car. This innovative study aims to assess the impact of attentional workload modulation on cerebral activity during a simulated driving task using magnetoencephalography (MEG). A car simulator equipped with a steering wheel, turn indicators, an accelerator and a brake pedal has been specifically designed to be used with MEG. Attentional demand has been modulated using a radio broadcast. During half of the driving scenarios, subjects could ignore the broadcast (simple task, ST) and during the other half, they had to actively listen to it in order to answer 3 questions (dual task, DT). Evoked magnetic responses were computed in both conditions separately for two visual stimuli of interest: traffic lights (from green to amber) and direction signs (arrows to the right or to the left), shown on boards. The cortical sources of these activities have been estimated using a minimum-norm current estimates modelling technique. Results show the activation of a large distributed network similar in ST and DT and similar for both the traffic lights and the direction signs. This network mainly involves sensory visual areas as well as parietal and frontal regions known to play a role in selective attention and motor areas. The increase of attentional demand affects the neuronal processing of relevant visual information for driving, as early as the perceptual stage. By demonstrating the feasibility of recording MEG activity during an interactive simulated driving task, this study opens new possibilities for investigating issues regarding drivers' activity.
\end{abstract}

\section{Introduction}

Despite a continuous decrease in deaths on the French roads during the last ten years, as in other countries, road safety remains a major priority for the government. It is well-known that human errors, whatever their sources, play an important part in car crashes (For example, McEvoy et al., 2006; Stutts et al., 2001; Young et al., 2003). Amongst these sources, attentional factors play a very important role. Indeed, driving is a complex task which requires sustained and selective attention. Drivers have to constantly adapt their behavior to the changing environment and anticipate future situations. Because of our attentional capacities, only a limited amount of information is processed in-depth at any one time. Therefore, safe and efficient behavior implies that drivers extract and process the most relevant pieces of information according to their objectives and previously-acquired knowledge, redirect their attention when facing novel potentially important events and ignore irrelevant sources of information.

In this context, even minor diversions of attention from the driving task can prove to be disastrous, particularly when a critical situation occurs. A recent American naturalistic study, recording the driving activity of more than 100 drivers for one year (Klauer et al., 2006), reports that, in $78 \%$ of all crashes and $65 \%$ of near-crashes, the driver was inattentive within the 3 seconds prior to the accident. In this study, driver inattention was induced by sleepiness or drowsiness, as well as secondary tasks or the fact of looking away from the road ahead. However, it can be useful to distinguish issues related to alertness (degree of arousal on the sleep-wake axis) from those related to selective attention since their impact on driver behavior and potential counter-measures can differ considerably. Deficits in selective attention processes are multiple and can occur even when drivers are completely alert. They can be due to dual activities that are voluntary (eating, drinking, phoning, smoking, reading...) or involuntary (being lost in thought, attention attracted by something inside or outside the vehicle, not looking at a relevant source of information) while driving and can induce physical and/or cognitive disruptions. Considering these attention deficits independently from the alertness issue, some epidemiological and naturalistic driving studies estimate that they are a major contributing factor in at least a quarter of all car collisions (Klauer et al., 2006; Stutts et al., 2005). Behavioral studies on simulator and on road have shown that driving performances deteriorate in the case of multitasking behind the wheel (for example, Horberry et al., 2006; Lansdown et al., 2004). Although the deleterious impact of physical activities (manipulating an object) on driving is easily understandable and identifiable, that of cognitive activities is less obvious. However, the use of a cell phone while driving has been shown to increase the risk of accidents, not only due to the physical manipulation it induces but also due to cognitive distraction (Bruyas et al., 2006; Horrey and Wickens, 2006; Strayer et al., 2003; Strayer and Johnston, 2001). In addition, cognitive distraction can occur without mobile phone conversation. Indeed, the increasing presence of assistance and information systems in cars, such as GPS, the possibility of listening to audio-books or the simple fact of thinking about personal issues when driving are only a few examples of activities implying cognitive distraction in a more or less intense way. Therefore, it seems important to gain a better 
understanding of the impact of cognitive distraction on drivers. Until now, the impact of attentional defects on driving has mainly been assessed by studying behavioral performances, naturalistic observations or self-reports (Lee, 2008). These approaches can be very informative but they only provide information about the observable or self-reconstructed impact of attentional defects. In order to successfully interact with the environment, sensory data have to be perceived, processed and interpreted to lead to pertinent and observable behavioural responses. Neuroimaging techniques can help to better understand these different steps. They can bring new information to the investigation of specific attentional defects on driving performance by examining the different cerebral stages of information processing.

Electroencephalography and magnetoencephalography (EEG and MEG) are particularly adapted for this purpose. Indeed, the analyses of the event related potentials (ERP) or evoked magnetic fields coming from these techniques enable to characterize the dynamics and spatial distribution of brain activity induced by the perception and the processing of a particular event with an excellent time resolution (fractions of amillisecond) (Luck, 2005). These pieces of information enable to examine the different stages of information processing and allocation of attention. By comparing the brain activity according to the attentional demand of the experimental task, these techniques provide information about when, where and how attention can impact the information processing.

For instance, Garcia-Larrea et al. (2001) have observed the impact of mobile phone use on a simple visual reaction task. Their results show that the increase in attentional demand by phone conversation does not delay target detection times but decreases attentional allocation and interferes with motor preparation processes. Using a more complex task - visual search task, Gherri and Eimer (in press) have also observed an impact of a verbal dual task on ERP components from the perceptual stage.

Until now, only a few researchers have used EEG in the more specific domain of driving. Their main purpose was to assess the impact of an increase in attentional demand on the processing of task-relevant information (brake light of the previous car) (Bruyas et al., 2006; Strayer and Drews, 2007) or task irrelevant information (arbitrary sounds in the environment) (Raabe et al., 2005; Rakauskas et al., 2005; Wester et al., 2008). Attentional demand modulation was induced either by a conversation (with a passenger or via a mobile phone) or by the driving task to perform (free driving or following a vehicle). These studies mainly analyzed the characteristics of the ERP component P3. Consistent with Garcia-Larrea et al. (2001), they found a decrease of P3 amplitude following an increase in attentional demand, suggesting a deterioration of the capacity to process taskrelevant stimuli. However, their analyses only concerned 3 or 4 sensors and did not allow for an in-depth examination of the spatial distribution of this activity.

Although EEG is easier to use in a driving simulator than the more cumbersome MEG, the latter is more efficient in estimating spatial distributions of the sources owing to the minimal distortion of the signal. In addition, the best signaltonoise ratio at the level of the sources enables to perform a time-frequency analysis at the level of the sources and an analysis of causality that can shed light on brain functional connectivity. However, MEG is much more expensive and, in the context of driving, implies setting up a car simulator that is adapted to this specific environment. These reasons may explain why it has only been used once in this context. This study was carried out by Bowyer et al. (2009) who investigated the impact of a conversation on a visual detection task (red light) while watching a driving video. They observed that the conversation induced an increase in reaction times to the visual event, correlated with a decrease in brain activation response variation in the following two regions that are important for the integration of visual information and motor response: the right superior parietal lobe (BA 7) also involved in orientation of attention and multitasking and the visual area (BA 19). However, in this study, subjects could not interact with the driving scene, and the red light was not related to the driving scene.

Here, we report an innovative MEG experiment using an interactive car simulator to examine the impact of an increase in attentional demand on the neuronal processes involved in processing a relevant driving visual cue. Participants were instructed to drive in a simulated urban driving environment as they would normally do. In particular, they had to brake at amber lights (in order to stop at the red lights) and to follow direction signs using the turning indicator appropriately. During the whole experiment, radio broadcasts were presented to the participants. Two experimental conditions were designed in order to modulate the attentional demand: in the simple task, subjects could ignore the radio, and in the dual task, they had to listen actively to the radio in order to answer some questions (see the Experimental procedure section for further details).

We hypothesized that the increase in attentional demand would correlate with a modulation of activity in attentional networks and in visual areas. In particular, we expected to observe modulation of brain activity in two partially segregated brain networks that are known to interact to determine where and what we attend to. First, in accordance with Bowyer et al. (2009), we expected a modulation of activity in a dorsal fronto-parietal network (centered on the posterior parietal cortex and the frontal eye field) which contributes to the selection of sensory stimulus based on internal goals or expectations and links them to appropriate motor responses (top-down control of attention). In addition, we expected a modulation of activity in a right ventral fronto-parietal network (centered on the temporo-parietal junction and the inferior and middle frontal gyri) known to contribute to the detection of unattended or low frequency events, independent of their location, sensory modality 
or response demands (bottom-up control of attention) (For review see Bressler et al., 2008; Corbetta et al., 2008; Corbetta and Shulman, 2002). Our results were partly presented in Fort et al. (2010).

\section{Results}

\subsection{Behavioral results}

As the distribution of the reaction times to a stimulus departs from the Gaussian distribution, we used the LATER model (linear approach to threshold with ergodic rate) (Carpenter and Williams, 1995; Sinha et al., 2006) to analyze the time required to make a decision. This model considers that the response is triggered when the evidence - starting from an initial level S0 - reaches a decision threshold level ST which is linked to the complexity of the task. This evidence accumulates at a constant rate $\mathrm{r}$ between S0 and ST that follows a Gaussian distribution allowing a variance analysis (see Experimental procedures). As expected since the complexity of each stimulus (traffic light and arrow) is constant, the variance analysis on the threshold difference (ST-S0) showed no significant difference between simple task (ST) and dual task (DT) (F=1.845; $\mathrm{p}=0.197)$.

Fig. 1 presents the $\mathrm{r}$ calculated for traffic lights and arrows separately. The distribution of the time required for making a decision concerning the traffic lights shows the presence of few early responses whereas only one main distribution is observed for the arrows. The variance analysis has been done only on the main distribution. Concerning the traffic lights, it revealed that the time required for making the decision (r) is significantly different between ST (399 ms) and DT (430 ms) $(\mathrm{F}=8.167 ; \mathrm{p}=0.013)$. This time difference corresponds to a difference in braking distance of $0.42 \mathrm{~m}$ between ST and DT. For the occurrence of the arrows on direction boards, there is no significant difference between the conditions (ST/DT) with the time required for making the decision ( $\mathrm{r})$ of $890 \mathrm{~ms}$ in ST and 875 in DT $(\mathrm{F}=2.301 ; \mathrm{p}=0.153)$.

Note that all participants properly followed all direction signs and stopped at all amber traffic lights in ST and DT. Concerning the performance in the DT, participants gave $77 \%$ of correct answers to the questions about the radio broadcasts. In addition, no correlation was observed between reaction time and the time during the experiment ( $\mathrm{r}=0.08$ for traffic lights and $\mathrm{r}=0.01$ for arrows) suggesting that there are no fatigue or learning effects.

\section{2. $M E G$ results}

MEG data have been analyzed using a distributed model, the minimum-norm-estimation method (MNE) (Hämäläinen and Ilmoniemi, 1994). This model consists in estimations by modeling the brain activity (source and time-course) specifically induced by the stimuli of interest using an average locked to the events. This method selects the most plausible source distribution among those compatible with the measurements without explicit a priori information on the source. The neuronal sources of MEG activity have been estimated separately for the change of traffic lights (green to amber) and for the occurrence of the arrows on direction boards in both ST and DT conditions. For the purpose of simplicity, only the major activities observed (amplitude superior to $20 \mathrm{pA} \mathrm{m}$ with a stable spatial distribution for at least $20 \mathrm{~ms}$ ) will be described.

\subsubsection{Neuronal network activated by the change of traffic lights from green to amber}

The results show the activation of a widely distributed neuronal network in ST and DT (Fig. 2).

As depicted in Fig. 2A, a first large source of activity was observed within the primary and secondary visual areas from 140 $\mathrm{ms}$ to the end of the analysis window (Brodmann areas, BAs 17/18 and 19). This activity was maximal around $230 \mathrm{~ms}$ and was larger in the left hemisphere both in ST (amplitude of the max on this region: $46 \mathrm{pA} \mathrm{m}$ ) and DT (58 pA m).

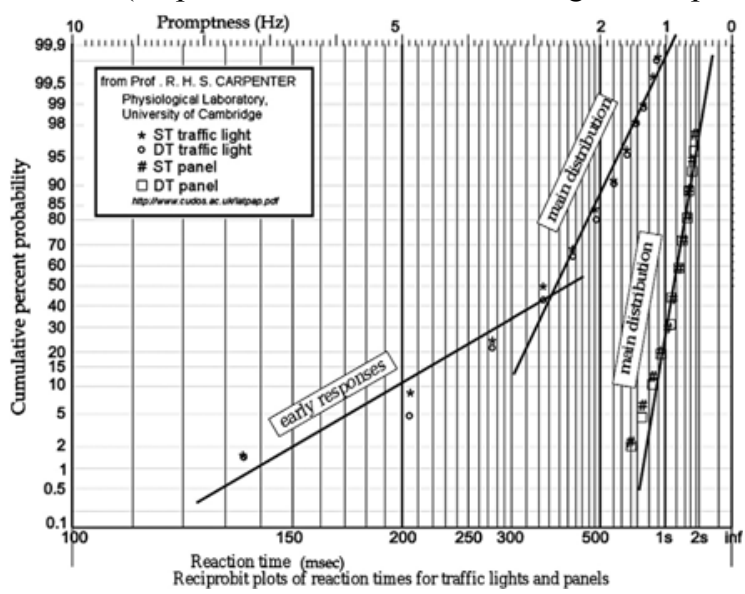

Fig. 1 - Cumulative percent probability of reaction times on a probit scale vs reaction times, for traffic lights and boards in both simple and dual task conditions (ST and DT). 


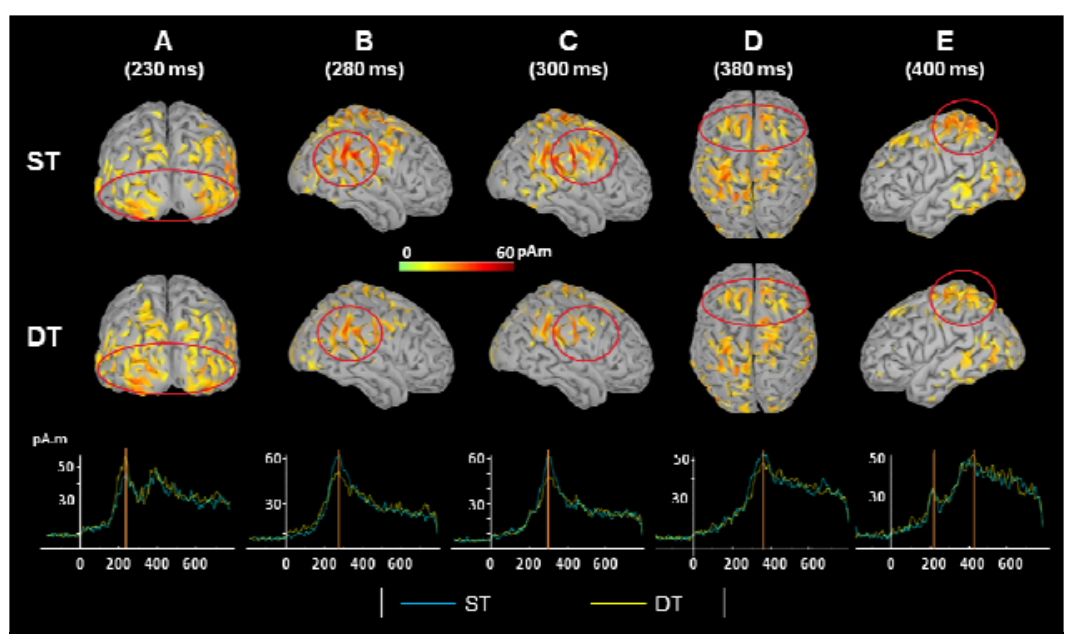

Fig. 2 - MNE results showing the main neuronal network activated by (A) the traffic light change from green to amber, in simple task (ST) and dual task (DT) according to the time rendered onto three different views (lateral, posterior and horizontal) of a standard template brain and time-course estimations of the activity in surrounding brain regions

A strong activity was observed around the right temporoparietal junction (BA 39) from 200 ms (Fig. 2B). This activity reached its maximum around $280 \mathrm{~ms}$ in both ST $(64 \mathrm{pA} \mathrm{m})$ and DT $(51 \mathrm{pA} \mathrm{m})$. Then, it spreads out to the posterior areas of the right frontal lobe (BAs 4 and 6) (Fig. 2 C). This activity started around $200 \mathrm{~ms}$ and reached its maximumaround $300 \mathrm{~ms}$ in ST (62 pA m) and $325 \mathrm{~ms}$ in DT (49 pA m).

The bilateral medial frontal areas (BA 6, supplementary motor areas) were activated with a maximum around 360 ms in ST and DT (48 vs 42 pA m) (Fig. 2D).

Finally, the bilateral superior parietal areas (BA 7) were also activated (Fig. 2E). In ST, this activity first occurred on the left hemisphere from $170 \mathrm{~ms}$ with two peaks: one at $215 \mathrm{~ms}$ in ST (32 pA m) as in DT (34 pA m) and one at $400 \mathrm{~ms}$ in ST (50 $\mathrm{pA} \mathrm{m})$ and at $430 \mathrm{~ms}$ in DT (53 pA m). The right superior parietal cortex was also activated, but less so, with one peak at $285 \mathrm{~ms}$ in ST (43 pA m) and another at $335 \mathrm{~ms}$ in DT (35 pA m).

\subsubsection{Neuronal network activated by direction board processing}

Source analysis revealed the activation of a similar neural network activated by the occurrence of the arrows on the direction boards (Fig 3). Indeed, a strong activity was observed within the visual areas mainly in the left hemisphere with a peak of activity at $215 \mathrm{~ms}$ (amplitude of the max on this region: $57 \mathrm{vs} 56 \mathrm{pA} \mathrm{m}$ in ST and DT, respectively) (Fig. 3A). Then, the right temporoparietal junction was activated with a maximum around $315 \mathrm{~ms}$ in ST (45 pA m) and around $340 \mathrm{~ms}$ in DT (39 pA m) (Fig. 3B). The median frontal area was activated with a peak around $340 \mathrm{~ms}$ in ST as in DT (33 vs $24 \mathrm{pA} \mathrm{m})$ (Fig. 3C). The bilateral medial frontal areas were also activated, but less so than for the traffic lights (Fig. 3D). This activity reached its maximum around $360 \mathrm{~ms}$ in ST and $420 \mathrm{~ms}$ in DT (35 and $25 \mathrm{pA} \mathrm{m}$, respectively). Finally, the left parietal cortex (BA 7) was activated with two peaks: one around $215 \mathrm{~ms}$ in ST and DT (27 and $18 \mathrm{pA} \mathrm{m}$, respectively) and one around 380$390 \mathrm{~ms}$ in ST (34 pA m) and $395 \mathrm{~ms}$ in DT (30 pA m) (Fig. 3E). 


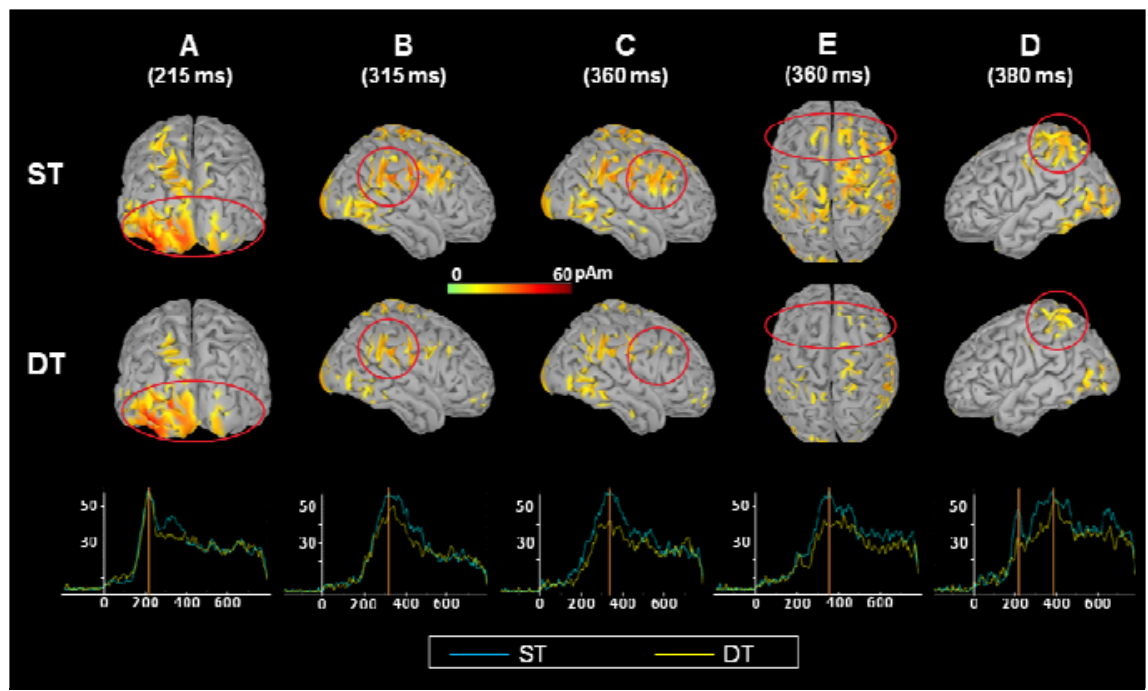

Fig. 3 - MNE results showing the main neuronal network activated by the occurrence of arrows on direction boards, in simple task (ST) and dual task (DT) according to the time rendered onto three different views (lateral, posterior and horizontal) of a standard template brain and time-course estimations of the activity in surrounding brain regions.

The comparison of this network with that elicited by traffic lights using a Student t-test showed some significant differences in the time-courses of activity in all regions. This can be explained either by latency differences or a damping of activity between both conditions. Because of these differences that can be due to the physical differences between both stimulations and the different responses that they induce (see Discussion), we realized a separate analysis of both stimuli.

\subsubsection{Impact of the dual task on traffic light processing}

In order to determine the impact of a change in drivers' attentional demand, the MEG activity estimated by the MNE method in ST and DT was compared with a Student t-test $(\mathrm{p}=0.001)$. This comparison shed light on a wide neural network including the areas cited above as well as the other brain areas involved in the processing of attention (Fig. 4).

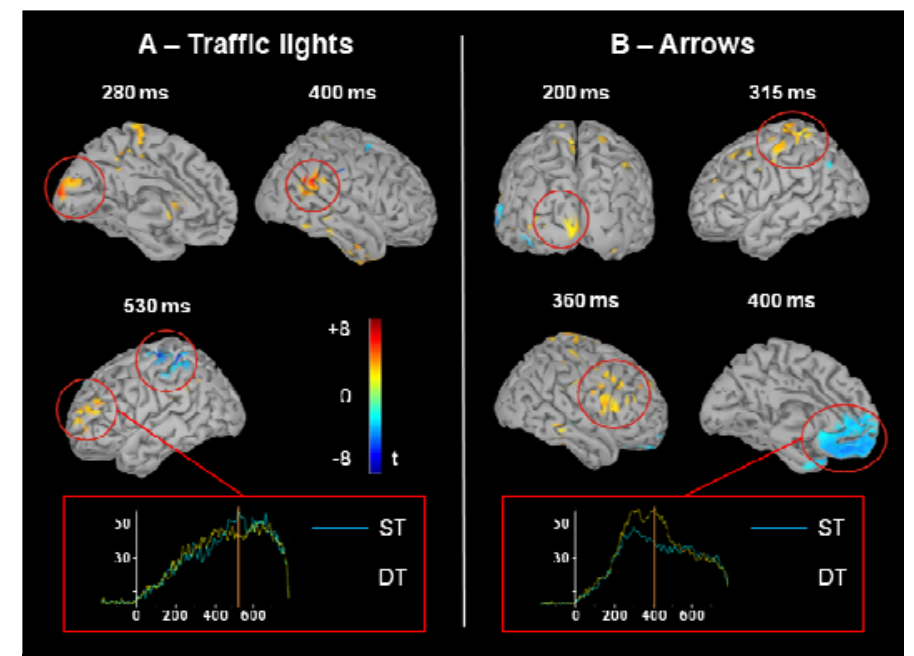

Fig. 4 - Statistically significant differences (t-test corrected for multiple comparisons) between simple task (ST) and dual task (DT) according to the time ([ST]-[DT]) separately for (A) the traffic light change from green to amber and (B) the occurrence of arrows on direction boards. Time-course estimations of the activity are depicted for the following two brain regions that have not been described in the main network: the left dorsolateral prefrontal area for traffic lights and the frontopolar area for arrows. 
The differences in amplitude observed here appear at some latencies but without univocal correspondence with any peak described above. They can be induced either by a damping of activity in these regions or by timing differences in the timecourses of these activities.

This analysis revealed that the activity was significantly weaker in DT than in ST in the primary visual area in medial occipital area (BAs 17 and 18) from 270 to $300 \mathrm{~ms}$ (amplitude at $290 \mathrm{~ms} 24$ pAm and 20 pAm in ST and DT, respectively).

The activity is also significantly weaker in DT than in ST in the right temporo-parietal junction (BA 39; $\mathrm{x}=59, \mathrm{y}=-54, \mathrm{z}=13$ ) from 395 to $430 \mathrm{~ms}$ (amplitude at $400 \mathrm{~ms}$ : 33 and 22 pAmin ST and DT, respectively) and in the left dorsolateral prefrontal area (BAs 10 and 46; $\mathrm{x}=-46, \mathrm{y}=33, \mathrm{z}=35$ ) from 520 to $530 \mathrm{~ms}$ (amplitude at $525 \mathrm{~ms}$ : 28 and $21 \mathrm{pA} \mathrm{m}$ in ST and DT, respectively).

Finally, one area presents significantly a stronger activity in DT than in ST, the left parietal area $(B A 7 ; x=-29, y=-53$, $\mathrm{z}=56$ ) from 520 to $535 \mathrm{~ms}$ (amplitude at $525 \mathrm{~ms}$ : 32 and $41 \mathrm{pAm}$ in ST and DT, respectively).

\subsubsection{Impact of the dual task on direction board processing}

Significant differences between ST and DT in the neural processing of the arrow occurrences on the direction boards were observed in several areas (Fig 2B).

The activity in the left occipital visual areas (BAs 17 and $18 ; \mathrm{x}=-4, \mathrm{y}=-101, \mathrm{z}=12$ ) is significantly weaker in DT than in ST from 190 to $220 \mathrm{~ms}$ (amplitude at $210 \mathrm{~ms}$ : 45 and 37 pAmin ST and DT, respectively). The left parietal area (BA 7; x=-29, $\mathrm{y}=-53, \mathrm{z}=56$ ) also presents a weaker activity in DT than in ST from 280 to $320 \mathrm{~ms}$ (amplitude at $300 \mathrm{~ms}: 26$ and 19 pAmin ST and DT, respectively), as the right middle frontal areas (BA 9/44; $x=37, y=3, z=40$ ) from 350 to 380 ms (amplitude at $360 \mathrm{~ms}: 32$ and $20 \mathrm{pAm}$ in ST and DT, respectively). One region only showed a significantly stronger activity in DT than in ST, the left medial orbito-frontal area (BA 10/11; $\mathrm{x}=-2, \mathrm{y}=48, \mathrm{z}=4$ ) from 355 to $430 \mathrm{~ms}$ (amplitude at 400 ms: 14 and 20 pAmin ST and DT, respectively).

\section{Discussion}

Using MEG, we investigated brain activity induced by the processing of the following visual stimuli relevant to the driving task: traffic lights turning fromgreen to amber and occurrences of arrows indicating the direction to follow. Then our analysis focused on the impact of an increase in attentional demand by comparing this activity according to the attentional state of the participants. Attentional demand was manipulated using a cognitivedual task inwhichthe participant had to listenactively to a radio broadcast. This task is interesting since it can be compared to a new activity observed on the road, listening to audio-books, for which the driver has to focus his/her attention continuously in order to follow the story.

Behavioral data revealed that our participants reacted much more quickly to the traffic light changes from green to amber than to the appearance of arrows on the boards. This can be due to the fact that the traffic light change to amber is an imperative signal meaning that you have to stop (no choice). On the other hand, the arrow is an informative signal meaning that, at the next intersection, you should turn right or left (choice between two answers). In addition, some people can choose to wait to be nearer to the intersection before operating the indicator. This can also explain why we did not find any early responses for the arrows. Moreover, we found a significant difference in the reaction time according to the attentional task demand for traffic lights but not for arrows. This again can be explained by the fact that RT are less stressed for arrows than for traffic lights. Consequently, the impact of the modulation of the attentional demand is less visible.

Concerning the main neuronal network involved in processing relevant visual cues for driving, the results revealed that, regardless of the attentional state of the subject and the kind of information to be processed (traffic light or arrow), it implies visual areas and a right fronto-parietal network. Primary and secondary visual areas showed stronger activity in the left hemisphere than in the right. This difference could be due to the partly crossed architecture of the visual streams. Indeed, as all traffic lights and arrows appear in the right field of view, they are firstly processed in the left cerebral hemisphere. The fronto-parietal network observed here is consistent with that described in literature and involved during tasks requiring attention orientation to relevant cues (Bowyer et al., 2009; Nobre, 2001). Thus, the temporo-parietal junction has been observed repeatedly in a variety of tasks requiring the redirection of attention to task-relevant information (ventral attention system) (Corbetta and Shulman, 2002; Mitchell, 2008) and the superior parietal lobule would be engaged in tasks involving the shift of attention, as is the case in our study (dorsal attention system) (Bowyer et al., 2009; Le et al., 1998; Vandenberghe et al., 2001). All activities recorded are consistent with the driving task, which strongly engages topdown and bottom-up attention and implies shifting attention between a great number of potential relevant cues for safe driving. Interestingly, the peaks of activity within the right temporo-parietal junction and the middle frontal areas (ventral attention system) appear later for the arrows than for the traffic lights. Again, this difference can be explained by the fact that, even if both pieces of information can be anticipated (when the green traffic light or the white board starts to be visible on the screen), the time pressure for traffic lights is stronger than for arrows. Then, an activation of the bilateral medial frontal areas which seem to be linked to the motor responses has also been observed. Nevertheless, this activity is stronger for traffic lights than for 
arrows. This difference can be explained by the difference in the time needed to make a decision according to the kind of cues. Behavioral results have shown that the mean reaction time to the appearance of arrows is very long compared to the reaction time to traffic light changes and it is longer than the analysis window. Consequently, brain activity linked to the motor responses for the arrows is not visible in this analysis. Note that another factor can influence this brain activity, the type of motor response related to each kind of visual information. Indeed, the motor response for the traffic lights consists in removing the foot from the accelerator pedal before pressing the brake pedal. Comparatively, the motor response for the arrows (consisting in activating the indicator) is easier. As the indicator is close to the wheel, this can be done with a simple finger movement. These results are consistent with a functional magnetic resonance imaging (fMRI) study examining the brain network involved in simulated car driving (Graydon et al., 2004). These authors also observed the activation of a fronto-parietal network including the bilateral posterior parietal cortex (BA 7), the right temporo-parietal junction (BA 39) and the middle frontal gyrus (BA 6) that would be related to the selection of sensory information and responses, as well as to the detection of behaviorally relevant sensory events (dorsal and ventral attention systems).

Concerning the impact of the task attentional demand, comparisons of activity induced by the processing of the traffic lights according to the condition demonstrated a modulation of activity in the main network described above as well as in two other brain regions. Hence, a decrease in brain activity in primary visual areas is observed (BAs 17 and part of 18). These results suggest a modification of the early visual processes by the increase of the demand of attention. Modulations of activity in attention neural network have also been observed. The dorsolateral prefrontal cortex (BA 46) showed lower activations in conditions of high attentional demand. This region may be involved in action selection and could play a specific role in the top-down control of neuronal activity in regions processing task-relevant representations (Rowe et al., 2005, Rowe et al., 2000). According to this, these results seem to reflect a diminution of top-down attention for the selection of relevant information (traffic lights) and action selection (to brake or not) in the dual task situation (Corbetta and Shulman, 2002). Nevertheless, the posterior parietal cortex that is also known to be part of the dorsal attention system showed stronger activity in conditions of high attentional demand. The reason for this activation can be twofold. First, it is well-known that the posterior parietal cortex plays a role in spatial operations by integrating spatial information from the various elements of the environment for navigation (For a review, see Calton and Taube, 2009). Furthermore, this region has been shown to be involved in attentional shifting. Consequently, the increase in the dual task condition can be due to the fact that subjects had to shift their attention between the radio broadcast and the driving scene (Vandenberghe et al., 2001). Finally, a diminution of activity is observed around the right temporo-parietal junction (BA 39), known to play a role in the detection of low frequency and complex events (ventral attention system) (Corbetta and Shulman, 2002; Mitchell, 2008). This result suggests a modulation of attention orientation to relevant events in conditions of high attentional demand and is in accordance with the difference in RT observed for the traffic light.

Concerning the occurrence of the arrows on direction boards, modulations of activities according to the subjects' attentional state have also been observed in several areas. Most of the following regions presented a stronger activity in the simple task than in the dual task: the occipital visual areas, the right frontal area, the left posterior parietal cortex, the left suggests a degradation of the processing of the direction boards because of the attentional share between the two tasks. In addition, the decrease of activity within the visual areas and the posterior parietal cortex is consistent with the data of Bowyer et al. (2009) obtained for the detection of a visual target while viewing a driving video. Only the left frontopolar cortex (BA 10/11) presented stronger activity in conditions of high attentional demand. This region would contribute to reasoning and problemsolving and would underlie decision-making. This region is also robustly engaged in multitasking behaviors (Burgess et al., 2005).

It is possible to question the origin of the effects observed in this task. Indeed, as subjects had to listen actively to the radio, the differences observed between the ST and the DT could be due, at least partially, to a main effect of the auditory task. Nevertheless, we think that this is not the case. Indeed, this auditory effect would probably consist in a sustained activity throughout thewhole experiment. This activity is then probably removed in the visual evoked response with the baseline correction. This hypothesis is reinforced by the fact that our analysis did not reveal any activity in the auditory areas.

Results from fMRI studies examining the impact of dual tasks on the activation of brain networks during simulated driving partly diverge. Just et al. (2008) observed decreased activation in the bilateral parietal cortex as well as in the occipital cortex with the addition of a listening task. On the other hand, Hsieh et al. (2009) found increased activity in the bilateral superior parietal lobe and in the inferior and middle frontal gyrus when drivers have a phone conversation. Further research is necessary to examine the impact, in more depth, of multitasking when driving and to have a better understanding of the complexity of these processes. The temporal advantage of MEG can help us to have a better understanding of this complex activity by analyzing the impact of attentional modulation step by step.

Furthermore, the kind of information to be processed seems to be an important factor to take into account. Indeed, in this experiment, the modulation of the task attentional demand has different impacts on the neuronal process linked to the different stimuli of interest. In addition to the physical difference between the two events discussed above, there are at least 
three possible and non-exclusive explanations for this differential effect. The first explanation concerns the choice of the answer. Subjects had to choose between two actions for the traffic lights - to brake or to continue - and 3 actions for the direction boards - to put the right or left indicator on or to do nothing. Moreover, reacting to the traffic lights by braking implied that the driver would do nothing for a few seconds but wait for the traffic lights to change from red to green. On the other hand, reacting to the occurrence of the arrow by putting on the indicator is the first step to managing a left or right turn that implies controlling the situation and the vehicle trajectory. This stronger difficulty of the task for the driver following the reaction to the arrow compared to the traffic light can explain (1) the stronger activation of the frontopolar cortex in the DT than in the ST for the arrow and (2) the stronger activation of the left posterior parietal cortex in the DT than in the ST for the traffic light. Indeed, for the traffic light, participants had to stop the vehicle and wait until the green light showed. In this condition in the DT, they could redirect their attention to the radio in order to find the answers to the questions. Finally, the kind of information driven by the two stimuli is different. In one case, the information has a safety and legal issue, as everyone knows the risk takenwhenpassing a red traffic light and the related interdict. On the other hand, the fact of missing a direction board has no legal or safety consequence. There is no vital risk induced by turning left when you should have turned right except that of being lost. Consequently the neuronal basis supporting the processing of these two kinds of stimuli can be influenced differently by the dual task situation. Further research is necessary to have a better understanding of the impact of these different factors.

To conclude, the question of the role played by attentional defects in car crashes is a topic of increasing concern in road safety. This study shows that increasing the attentional demand while driving affects the neuronal processing of relevant visual information for driving, as early as the perceptual stage. Moreover the effect observed here differs according to the kind of information to be processed. This result calls for an investigation of the impact of attentional defects in taking into account the object to which attention is directed. As some impact of the dual task has been observed on motor preparation processes during simple visual tasks (Garcia-Larrea et al., 2001), further analysis should be carried out in order to investigate this issue in the context of driving. In addition, a time-frequency analysis would also enable to examine more continuous activity and a connectivity analysis would be very informative for gaining amore detailed vision of the brain processes involved in our task and to allow deeper interpretations. By demonstrating the feasibility of recording MEG activity during an interactive simulated driving task, this study opens new possibilities for investigating issues related to drivers' activity.

\section{Experimental procedures}

\subsection{Participants}

Thirteen right-handed male volunteers, $21-28$ years old (mean age $25.4 \pm 2.1$ ), were included in the analysis described here. They had no history of neurological disorders and normal or corrected-to-normal vision (contact lenses). All participants were native French speakers, had held a driving license for at least $3 \mathrm{y}$ and drove at least $3000 \mathrm{~km} / \mathrm{y}$. Participants gave their informed consent in accordance with the Code of Ethics of the World Medical Association (Declaration of Helsinki) and were compensated for their participation.

\subsection{Stimuli and procedure}

Subjects drove in a car simulator adapted to the MEG environmental constraints to allow the recording of brain activity during the driving task. The simulator was equipped with a steering wheel, a turning indicator, an accelerator and a brake pedal. An interactive visual scene of an urban driving environment was projected onto a screen. Participants had to drive on single or dual carriageways, with little traffic and few pedestrians on the pavements. The subjects were seated at a distance of $87 \mathrm{~cm}$ in front of the screen (with a visual angle of $34^{\circ}$ ) and held the wheel. Head motions were restricted by using a head stabilizer bladder. Instructions were given to drive as they would normally do, to respect all traffic signs and to follow direction signs (arrows to the left or to the right) until the end of the scenario. In particular, subjects were instructed to follow traffic light rules whereby they had to stop at amber lights and follow direction signs (arrows shown on a white board) using the turning indicator appropriately (Fig.5). 


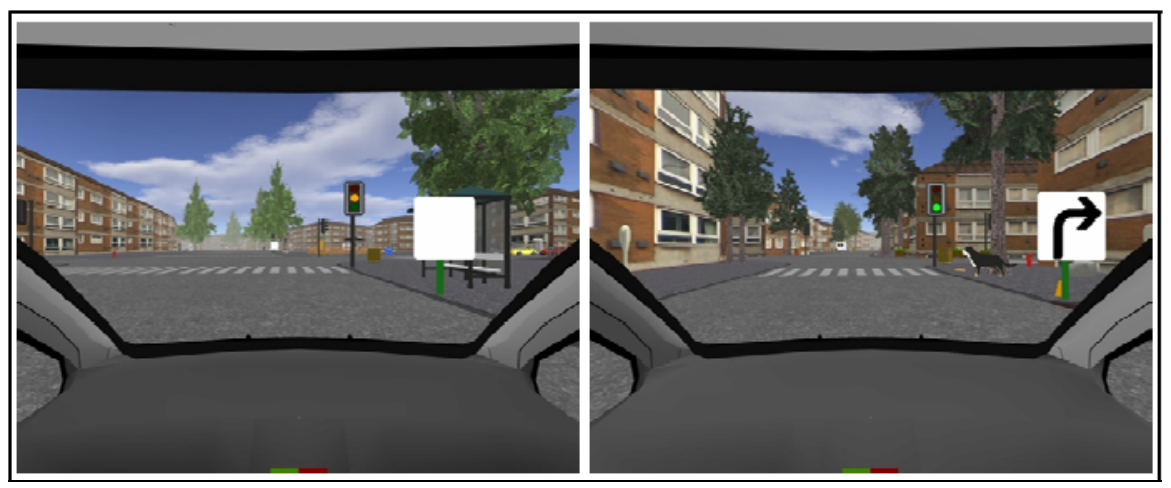

Fig. 5 - Screenshots illustrating the simulated environment with traffic lights and arrows

All traffic lights and direction signs were presented on the right-hand side of the road. Traffic lights could turn to amber when the participant's vehicle arrived at $31 \mathrm{~m}$ from the traffic light, otherwise it stayed green and the participant continued on his/her way (visual angle of the traffic light when it turns to amber: $0.5^{\circ}$ ). Concerning the direction signs, the arrow appeared on the white board when the participant's vehicle arrived at $27 \mathrm{mfrom}$ the board, otherwise the direction boards stayed white and the participant was to continue straight on (visual angle of the board when the arrow appears: $1.5^{\circ}$ ). Only one stimulus of interest could be given (traffic light change or arrow) at one given time. In other words, at one particular intersection, either participants had to drive straight on without stopping (no stimulus at all) or they had to stop at the traffic lights or follow a direction sign.

Firstly, participants were introduced to the simulator and drove one or two 5-min practice drives to familiarize themselves with the vehicle control and the visual environment during the experiment. Following this, participants drove in 18 different, randomly-controlled, driving scenarios, each lasting about 5min. During the experiment, 100 traffic lights turning from green to amber (30\% of all traffic lights) and 130 arrows (65 to the left and 65 to the right, $48 \%$ of all direction boards) were presented (i.e. 5.5 traffic lights and 7.2 arrows per scenario on average) ${ }^{1}$. For half of the scenarios (9), subjects were presented with radio broadcasts (comfortable sound level) which they were instructed to ignore at first - simple task (ST). In the other 9 scenarios, subjects had to listen actively to the broadcast in order to answer three questions asked beforehand dual task (DT). Questions were asked again at the end of each driving trial and responses were recorded in order to check if subjects observed the instructions correctly (Combe-Pangaud and Jacquet-Andrieu, in press). The order of the ST and DT conditions was intermixed. Subjects could take short breaks (1-3min) as necessary between blocks to minimize tiredness and eye movements and a longer break (10-15 min) was imposed on all participants in the middle of the experiment.

\subsection{Behavioral analysis}

Reaction times (RT) to the amber traffic lights and to the appearance of the arrows on the boards were recorded. RT to traffic lights correspond to the first action on the pedals that is the moment the participants released the accelerator pedal (before braking) and RT to arrows correspond to the moment when participants operated the turning indicator.

Whatever the experiment, the distribution of RT to a stimulus departs from the Gaussian distribution. Therefore, when plotting the reaction times as a conventional histogram, the result appears as a skewed distribution with a longer tail on the long RT side. For this reason, sets of transformations of distributions have been specifically investigated to analyze the reaction times more accurately. Here, we used the LATER model (linear approach to threshold with ergodic rate) (Carpenter and Williams, 1995; Sinha et al., 2006). In this model, we consider that the response is triggered when the evidence starting from an initial level S0 - reaches a decision threshold level ST. This evidence accumulates at a constant rate $\mathrm{r}$ between S0 and ST. From trial to trial, $r$ varies randomly as a Gaussian distribution with mean $\mu$ and variance $\sigma 2$. Since RT and $\mathrm{r}$ are reciprocally related to each other $(\mathrm{r}=1 / \mathrm{RT})$, we can perform a statistical analysis on $\mathrm{r}$. This model is also useful to detect early responses which induce a second mode (a second process) in the RT distribution. This is perfectly clear on the cumulative histogram of $r$ on a probit scale (see Fig. 5). In this case, there should be one straight line for a single process and two crossing lines for a dual brain process.

In our driving task, since the complexity of each stimulus (traffic light and arrow) is constant, we can hypothesize that the difference between the initial state and the decision threshold is constant. The mean and standard deviation of the distribution of speed of decision-making $\mathrm{r}$ can be estimated from RT, for each subject and each condition, from the probability density function:

\footnotetext{
${ }^{1}$ We chose to present fewer traffic lights because, as participants had to stop at the red light, they extended the duration of the experiment.
} 


$$
f_{r}(t ; \mu, \sigma)=\frac{1}{t^{2} \sqrt{2 \pi \sigma^{2}}} e^{-\frac{(\mu t-1)^{2}}{2 \sigma^{2} t^{2}}} \quad \text { pour } t \neq 0
$$

This distribution is continuous and defined along the whole time axis. To estimate the $f \hat{\mathrm{E}}$ and $f Đ$ parameters, an algorithm for non-linear curves fitting based on trust-regions has been used ${ }^{2}$. The weight method of the residuals used here is the LAR (least absolute residuals) method. This method finds a curve that minimizes the absolute difference of the residuals rather than the squared differences. Therefore, extreme values have less influence on the fit.

\subsection{MEG acquisition and analysis}

The experiment was conducted at the MEG Centre of CERMEP (Bron, France).

First, head coils were placed on the nasion and on left and right pre-auricular points prior to scanning for continuous head localization recording. Then, the location of these coils and the head-shape of each participant were digitized with Polhemus (Polhemus Inc., Vermont, USA).

MEG recording was performed using a whole-head MEG system (Omega 275, CTF, VSM MedTech Ltd.) with 275 radial gradiometers over the scalp and 33 reference channels for ambient field correction. Signals were digitized at a sampling rate of $300 \mathrm{~Hz}$ and were recorded continuously applying bandpass filtering from 0 to $75 \mathrm{~Hz}$. Vertical and horizontal eye movements (electrooculogram, EOG) were recorded for artefact control. A trigger marked the onset of each stimulus of interest (traffic lights turning from green to amber and occurrence of an arrow ondirectionboards) for trial extraction fromthecontinuous MEG data. Trials were rejected offline for excessive eye blinks, eye movements, head movements, muscular or electromagnetic artifacts. Therefore, epochs of MEG signal exceeding 4.10.12T and those for which head movements exceeded $8 \mathrm{~mm}$ on the 3 coils were automatically removed. For blink rejection, we first selected a few blinksmanually in order to create a template. This template was then used to look for all blinks and marked them automatically. The analysis was done with epochs for which no blink was present in the time window from $300 \mathrm{~ms}$ prestimulus to $900 \mathrm{~ms}$ post-stimulus. For trials with correct responses, the averaging epoch was defined from $200 \mathrm{~ms}$ before stimulus onset to $800 \mathrm{~ms}$ after stimulus onset, and data were baseline-corrected on the basis of a $200 \mathrm{~ms}$ pre-stimulus interval. After rejection of artifacts and omissions, an average of 39 traffic lights per subject in ST andDTand 57 direction boards per subject inST andDTwas kept for the analysis.

Source-space activity was estimated for each time point in each condition and participant for the two visual stimuli of interest (traffic lights fromgreen to amber and arrow to the right or to the left on direction boards), using a distributed model: the minimum-norm-estimation method (MNE). The MNE is an inverse modeling technique, which estimates cortical generator structures without any a priori assumptions regarding the location and/or number of current sources. This inverse source modeling and the consecutive statistical analysis thereof were conducted with the BrainStormR Toolbox (freely available at: http://neuroimage.usc.edu/brainstorm/).

In this study, anatomical MRIs of the participants were not recorded. For this reason, the individual cortical mesh of each participant was obtained by an affine transformation of a standard template (Colin27) to the head-shape of each subject. This head-shape was obtained by 3D scanning of the scalp using Polhemus. As the results were analyzed at group level, individual MRIs are not necessary.

The differences between ST and DT were estimated in a group analysis, over each point of the cortical mesh, using a Student t-test with permutations, which avoid assumptions about theoretical probability distribution (Pantazis et al., 2005). A post hoc correction for multiple comparisons (maximum statistic) was realized for each time point in the space of the cortical mesh (spatial dimension correction) and only the brain areas showing a stable statistical difference between the two conditions for at least $15 \mathrm{~ms}$ on a stable area will be described (time dimension correction). This time window of $15 \mathrm{~ms}$ is equivalent to $\mathrm{p}=0.001$ (Bonferroni correction). Locations of these main differences are reported using Talairach coordinates.

\section{Acknowledgments}

This work is part of the "DACOTA" project (ADEME 0566C0176, ANR-05-PDIT-004-02; PREDIT-GO 4 Technology for Security) which was granted by the French National Agency for Research. We would like to thank all the partners of this study: Thierry Bellet, Laurie Brun, Anne Buenerd, Andre Chapon, Antoine Clarion, Christian Collet, Joel Colloc, Andre Dittmar, Nicolas El Sayegh, Patricia Faure, Louis Frecon, SandraGatt, AymericGuillot, Nady Hoyek, Didier Leblanc, Florent Lebon, Francoise Lecaignard, Annick Maincent, Pierre Mauchand, Richard Meffre and Carolina Ramon.

\section{References}

\footnotetext{
${ }^{2}$ http://www.mathworks.com/help/toolbox/optim/ug/brnoxzl.html
} 
Bowyer, S.M., Hsieh, L., Moran, J.E., Young, R.A., Manoharan, A., Liao, C.-c.J., Malladi, K., Yu, Y.-J., Chiang, Y.-R., Tepley, N., 2009. Conversation effects on neural mechanisms underlying reaction time to visual events while viewing a driving scene using MEG. Brain Res. 1251, 151.161.

Bressler, S.L., Tang, W., Sylvester, C.M., Shulman, G.L., Corbetta, M., 2008. Top-down control of human visual cortex by frontal and parietal cortex in anticipatory visual spatial attention. J. Neurosci. 28, 10056.10061.

Bruyas, M.-P., Chapon, A., Lelekov-Boissard, T., Letisserand, D., Duraz, M., Aillerie, I., 2006. Evaluation de l'impact de communications vocales sur la conduite automobile. Rech. Transports Securite. 91, 99.119.

Burgess, P.W., Simons, J.S., Dumontheil, I., Gilbert, S.J., 2005. The gateway hypothesis of rostral prefrontal cortex (area 10) function. In: Duncan, J., et al. (Ed.), Measuring the Mind: Speed, Control, and Age. Oxford University Press; Oxford, pp. 217-248.

Calton, J.L., Taube, J.S., 2009. Where am I and how will I get there from here? A role for posterior parietal cortex in the integration of spatial information and route planning. Neurobiol. Learn. Mem. 91, 186-196.

Carpenter, R.H.S., Williams, M.L.L., 1995. Neural computation of log likelihood in the control of saccadic eye movements. Nature, $377,59-62$.

Combe-Pangaud, C., Jacquet-Andrieu, A., in press. Self-assessment, questionnaires and memory tests in a simulated driving task. First International Conference on driver distraction and Inattention, Göteborg (Sweden).

Corbetta, M., Shulman, G.L., 2002. Control of goal-directed and stimulus-driven attention in the brain. Nat. Rev. Neurosci. 3, $201-215$.

Corbetta, M., Patel, G., Shulman, G.L., 2008. The reorienting system of the human brain: from environment to theory of mind. Neuron 58, 306-324.

Fort, A., Martin, R., Daligault, S., Jacquet-Andrieu, A., Delpuech, C., 2010. Attention and processing of relevant information while simulated driving: a MEG study. Advances in Biomagnetism - BIOMAG2010, IFBME Proceedings, Dubrovnik, Croatia.

Garcia-Larrea, L., Perchet, C., Perrin, F., Amenedo, E., 2001. Interference of cellular phone conversations with visuomotor tasks: an ERP study. J. Psychophysiology. 15, 14-21.

Gherri, E., Eimer, M., in press. Active listening impairs visual perception and selectivity: an ERP study of auditory dual-task costs on visual attention. Journal of Cognitive Neuroscience.

Graydon, F.X., Young, R., Benton, M.D., Genik II, R.J., Posse, S., Hsieh, L., Green, C., 2004. Visual event detection during simulated driving: identifying the neural correlates with functional neuroimaging. Transp. Res. F 7, 271-286.

Hämäläinen, M.S., Ilmoniemi, R.J., 1994. Interpreting magnetic fields of the brain: minimum norm estimates. Med. Biol. Eng. Comput. 32, 35-42.

Horberry, T., Anderson, J., Regan, M.A., Triggs, T.J., Brown, J., 2006. Driver distraction: the effects of concurrent in-vehicle tasks, road environment complexity and age on driving performance. Accid. Anal. Prev. 38, 185-191.

Horrey, W.J., Wickens, C.D., 2006. Examining the impact of cell phone conversations on driving using meta-analytic techniques. Hum. Factors 48, 196205 .

Hsieh, L., Young, R.A., Bowyer, S.M., Moran, J.E., Genik Ii, R.J., Green, C.C., Chiang, Y.-R., Yu, Y.-J., Liao, C.-C., Seaman, S., 2009. Conversation effects on neural mechanisms underlying reaction time to visual events while viewing a driving scene: fMRI analysis and asynchrony model. Brain Res. $1251,162-175$.

Just, M.A., Keller, T.A., Cynkar, J., 2008. A decrease in brain activation associated with driving when listening to someone speak. Brain Res. 1205 , 70-80.

Klauer, S.G., Dingus, T.A., Neale, V.L., Sudweeks, J.D., Ramsey, D.J., 2006. The impact of driver inattention on near-crash/crash risk: an analysis using the 100-car naturalistic driving study data. Virginia Tech Transportation Institute.

Lansdown, T.C., Brook-Carter, N., Kersloot, T., 2004. Distraction from multiple in-vehicle secondary tasks: vehicle performance and mental workload implications. Ergonomics 47, 91-104.

Le, T.H., Pardo, J.V., Hu, X., 1998. 4 T-fMRI study of nonspatial shifting of selective attention: cerebellar and parietal contributions. J. Neurophysiol. 79, $1535-1548$.

Lee, J.D., 2008. Fifty years of driving safety research. Hum. Factors 50, 521-528.

Luck, S.J., 2005. An introduction to the event-related potential technique. Cambridge: MIT Press.

McEvoy, S.P., Stevenson, M.R., Woodward, M., 2006. The impact of driver distraction on road safety: results from a representative survey in two Australian states. Inj. Prev. 12, 242-247.

Mitchell, J.P., 2008. Activity in right temporo-parietal junction is not selective for theory-of-mind. Cereb. Cortex 18, 262-271.

Nobre, A.C., 2001. The attentive homunculus: now you see it, now you don't. Neurosci. Biobehav. Rev. 25, 477-496.

Pantazis,D., Nichols, T.E., Baillet, S., Leahy, R.M., 2005. A comparison of random field theory and permutation methods for the statistical analysis of MEG data. Neuroimage 25, 383-394.

Raabe, M., Rutschmann, R.M., Schrauf, M., Greenlee, M.W., 2005. Neural correlates of simulated driving: auditory oddball responses dependent on workload. Las Vegas (Nevada, USA): HCI International.

Rakauskas, M.E., Ward, N.J., Bernat, E., Cadwallader, M., Patrick, C., De Waard, D., 2005. Psychophysiological measures of driver distraction and workload while intoxicated. Third International Driving Symposium on Human Factors in Driver Assessment, Training and Vehicle Design.

Rowe, J.B., Toni, I., Josephs, O., Frackowiak, R.S., Passingham, R.E., 2000. The prefrontal cortex: response selection or maintenance within working memory? Science 288, 1656-1660.

Rowe, J.B., Stephan, K.E., Friston, K., Frackowiak, R.S., Passingham, R.E., 2005. The prefrontal cortex shows context-specific changes in effective connectivity to motor or visual cortex during the selection of action or colour. Cereb. Cortex 15, 85-95.

Sinha, N., Brown, J.T.G., Carpenter, R.H.S., 2006. Task switching as a two-stage decision process. J. Neurophysiol. 95, 3146-3153.

Strayer, D.L., Drews, F.A., 2007. Multi-tasking in the automobile. In: Kramer, A.F., et al. (Ed.), Attention: From Theory to Practice. Oxford:Oxford University Press.

Strayer, D.L., Johnston, W.A., 2001. Driven to distraction: dual-task studies of simulated driving and conversing on a cellular telephone. Psychol. Sci. 12, $462-466$. 
Strayer, D.L., Drews, F.A., Johnston, W.A., 2003. Cell phone-induced failures of visual attention during simulated driving. J. Exp. Psychol. Appl. 9, $23-32$. Stutts, J., Reinfurt, D.W., Staplin, L., Rodgman, E.A., 2001. The role of driver distraction in traffic crashes. AAA Foundation for Traffic Safety.

Stutts, J., Feaganes, J., Reinfurt, D., Rodgman, E., Hamlett, C., Gish, K., Staplin, L., 2005. Driver's exposure to distractions in their natural driving environment. Accid. Anal. Prev. 37, 1093-1101.

Vandenberghe, R., Gitelman, D.R., Parrish, T.B., Mesulam, M.M., 2001. Functional specificity of superior parietal mediation of spatial shifting. Neuroimage 14, 661-673.

Wester, A.E., Böcker, K.B.E., Volkerts, J.C., Verster, J.C., Kenemans, J.L., 2008. Event-related potentials and secondary task performance during simulated driving. Accid. Anal. Prev. 40, 1-7.

Young, K., Regan, M., Hammer, M., 2003. Driver distraction: a review of the literature. Monash University-Accident Research Centre. 
